\title{
Prevalence of the Fibromyalgia Phenotype in Patients With Spine Pain Presenting to a Tertiary Care Pain Clinic and the Potential Treatment Implications
}

\author{
Chad M. Brummett, Jenna Goesling, Alex Tsodikov, Taha S. Meraj, \\ Ronald A. Wasserman, Daniel J. Clauw, and Afton L. Hassett
}

Objective. Injections for spinal pain have high failure rates, emphasizing the importance of patient selection. It is possible that detecting the presence of a fibromyalgia (FM)-like phenotype could aid in prediction, because in these individuals a peripheral injection would not address pain due to alterations in central neurotransmission. We undertook this study to test the hypothesis that patients who have spine pain meeting survey criteria for FM would be phenotypically distinct from those who do not.

Methods. We studied 548 patients diagnosed as having primary spine pain. All patients completed validated self-report questionnaires, including the Brief Pain Inventory, the PainDETECT questionnaire, the Hospi-

Preliminary data presented in the Best Abstracts session at the American Society of Anesthesiologists Annual Meeting, Chicago, IL, October 2011.

Supported by the NIH (National Institute of Arthritis and Musculoskeletal and Skin Diseases grant R01-AR-060392 to Drs. Brummett and Clauw) and the American Society of Regional Anesthesia and Pain Medicine (Chronic Pain Research grant to Dr. Brummett), with additional funding from the University of Michigan, Department of Anesthesiology.

Chad M. Brummett, MD, Jenna Goesling, PhD, Alex Tsodikov, PhD, Taha S. Meraj, BS, Ronald A. Wasserman, MD, Daniel J. Clauw, MD, Afton L. Hassett, PsyD: University of Michigan, Ann Arbor.

Dr. Brummett has received consulting fees from Purdue Pharma (less than $\$ 10,000)$. Dr. Clauw has received consulting fees, speaking fees, and/or honoraria from Pfizer, Forest Laboratories, Eli Lilly, Pierre Fabre, Cypress Biosciences, Wyeth, UCB, AstraZeneca, Merck, Johnson \& Johnson, Nuvo, Jazz, Abbott, and Cerephex (more than $\$ 10,000$ each) and has received research grants from Forest Laboratories, Pfizer, Cerephex, and Nuvo. Dr. Hassett has received consulting fees, speaking fees, and/or honoraria from Pfizer (less than $\$ 10,000)$ and research funding from Pfizer and Bristol-Myers Squibb.

Address correspondence to Chad M. Brummett, MD, University of Michigan Health System, Department of Anesthesiology, Division of Pain Medicine, 1500 East Medical Center Drive, 1H247 UH, Box 5048, Ann Arbor, MI 48109. E-mail: cbrummet@umich.edu. Submitted for publication April 29, 2013; accepted in revised form August 27, 2013. tal Anxiety and Depression Scale, measures of physical function, and the FM criteria and severity scales.

Results. Forty-two percent of the patients were FM positive according to the FM criteria and severity scales. Compared with FM-negative patients, FMpositive patients were more likely to be younger, unemployed, and receiving compensation for pain and to have greater pain severity and pain interference and more neuropathic pain descriptors as well as higher levels of depression and anxiety and a lower level of physical function $(P<0.002$ for each comparison). Female sex, neuropathic pain, pain interference, and anxiety were independently predictive of FM status in a multivariate analysis $(P<0.01$ for all variables). Receiver operating characteristic curve analysis showed a strength of association of 0.80 as measured by the cross-validated $\mathrm{C}$ statistic.

Conclusion. Using the FM criteria and severity scales, we demonstrated profound phenotypic differences in a population of patients with spine pain. Although centralized pain cannot be confirmed with a self-report instrument alone, the pathophysiology of FM may help explain a portion of the variability of responses to spine interventions.

Spine pain is one of the most common causes of disability in the world. It is estimated that $10-15 \%$ of the US population seeks care for low back pain each year (1). Second only to the treatment of joint pain, spine pain is considered the most expensive musculoskeletal condition; estimates exceed $\$ 140$ billion in annual lost wages and treatment costs $(1,2)$. Recently, there has been an explosion in the use of minimally invasive therapies for the treatment of spine pain. Between 1997 and 2006 in the Medicare population, facet joint interventions increased by $543 \%$ (3), and epidural steroid injections increased by $102 \%$ (4). These and other mini- 
mally invasive therapies have high failure rates, implying that patient selection may play a crucial role $(5,6)$.

Some patient risk factors predictive of poor outcomes from epidural steroid injections and facet joint interventions include long duration of pain, opioid consumption, previous spine surgery, younger age, increased pain sensitivity, depression, and anxiety (5,712). Similarly, pain in other locations, depression, catastrophizing, and somatization all have been described as predictors of lesser analgesic response from lower extremity joint arthroplasty (13). It is possible that this collection of patient risk factors can be explained by a common pathophysiologic mechanism. There is a growing appreciation of the importance of augmented central nervous system (CNS) processing of pain and other symptoms in several chronic pain states (14). Such states lack clear peripheral pathology and have been given specific names, including fibromyalgia (FM), irritable bowel syndrome, and interstitial cystitis (14-17). Arguably the best studied of these, FM, is characterized by widespread body pain and comorbid symptoms (e.g., fatigue, trouble thinking, depression) without apparent peripheral pathology. Instead, pain sensitivity and neuropathic pain symptoms have been associated with alterations in central neurotransmission (15,18-22).

Experimental pain testing and functional neuroimaging studies have shown that subsets of individuals with classically described "peripheral" pain conditions, such as osteoarthritis and rheumatoid arthritis, demonstrate patterns of augmented CNS pain processing similar to those seen in conditions like FM, and thus potentially have a component of "centralized pain" $(23,24)$. The few experimental studies conducted in spine pain support the same conclusion. Pain threshold has been shown to be a robust predictor of pain response and physical function (25), and functional magnetic resonance imaging in low back pain has demonstrated patterns of augmented central pain processing similar to those seen with FM (26). However, the frequency of "centralized pain" in a population of patients with spine pain is not known.

In 2011, FM criteria and severity scales (also known as survey criteria for FM) were introduced for use in clinical and epidemiologic studies (27). The FM criteria and severity scales rely on the completion of a self-report questionnaire and, like the American College of Rheumatology (ACR) preliminary diagnostic criteria introduced in 2010 (28), do not require a tender point examination (27). The aim of the present study was to determine whether the FM criteria and severity scales could differentiate patients with spine pain in terms of measures of pain, affect, and function. FM is rarely diagnosed in this population, and patients are generally treated as having pain that is predominantly or solely due to peripheral pathology of the spine. We hypothesized that patients characterized as FM positive according to the FM criteria and severity scales (27-29), which were used as a surrogate of centralized pain in this study, would report pain that is more neuropathic in nature and would have higher levels of pain, depression, anxiety, and disability than those not meeting FM criteria. The clinical implications of this would be that a brief self-report measure (e.g., the FM criteria and severity scales) could eventually be used to guide patient selection for various interventions based in part on underlying pain mechanisms.

\section{PATIENTS AND METHODS}

Approval was obtained from the Institutional Review Board of the University of Michigan, Ann Arbor. We included new patients (age $\geq 18$ years) presenting to the University of Michigan Back and Pain Center (Department of Anesthesiology) from November 2010 to March 2012. As previously described (30), all new patients presenting for treatment at our academic outpatient pain clinic complete an intake packet that includes validated self-report measures of pain, psychological status, physical function, and demographic information (including age, sex, race, ethnicity, marital status, employment status, and compensation for pain, as reported by the patient from a predefined list). A coversheet for the intake packet ex-

Table 1. Diagnostic codes included in the study*

\begin{tabular}{llrr}
\hline \multicolumn{1}{c}{ Location, diagnosis } & $\begin{array}{c}\text { ICD-9 } \\
\text { code }\end{array}$ & $\mathrm{n}$ & Percent \\
\hline Neck and upper extremities & 723.1 & 84 & 15.3 \\
$\quad$ Cervicalgia, neck & & & 2.2 \\
Facet arthropathy cervical or spondylosis & 721 & 12 & \\
$\quad$ without myelopathy cervical & 722.4 & 3 & 0.5 \\
Degenerative disc cervical & 723.4 & 7 & 1.3 \\
Radiculopathy cervical or cervical- & & & \\
$\quad$ radiculitis/neck & 721.1 & 1 & 0.2 \\
Spondylosis with myelopathy cervical & 722.81 & 2 & 0.4 \\
Postlaminectomy syndrome cervical & 723 & 3 & 0.5 \\
Spinal stenosis cervical & & & \\
Middle back & 724.1 & 26 & 4.7 \\
Thoracic spine-middle back & 722.11 & 0 & 0.0 \\
Herniated nucleus pulposus-thoracic & & & \\
Low back and lower extremities & 724.2 & 269 & 49.1 \\
Low back/lumbago & 722.52 & 11 & 2.0 \\
Degenerative disc lumbar & 721.3 & 13 & 2.4 \\
Facet arthropathy lumbar or spondylosis & & & \\
$\quad$ without myelopathy lumbosacral & 722.1 & 17 & 3.1 \\
Herniated nucleus pulposus lumbar & 724.4 & 30 & 5.5 \\
Radiculopathy thoracic/lumbar & 724.02 & 27 & 4.9 \\
Spinal stenosis lumbar & 722.83 & 32 & 5.8 \\
Postlaminectomy syndrome lumbar & 739.4 & 9 & 1.6 \\
Sacroiliac joint dysfunction & 720.2 & 2 & 0.4 \\
Sacroiliitis & - & 548 & - \\
Total & & & \\
\hline
\end{tabular}

* Spine pain was defined by the primary diagnosis assigned by the treating physician using the International Classification of Diseases, Ninth Revision (ICD-9) codes during the new patient encounter. 
Table 2. Univariate analyses of differences between the groups*

\begin{tabular}{lccc}
\hline & $\begin{array}{c}\text { FM-negative } \\
(\mathrm{n}=257)\end{array}$ & $\begin{array}{c}\text { FM-positive } \\
(\mathrm{n}=186)\end{array}$ & $P$ \\
\hline FM criteria and severity scales score & $8.46 \pm 2.79$ & $17.2 \pm 3.89$ & $<0.0001$ \\
Demographics & & & \\
Age, years & $51.9 \pm 17.5$ & $46.9 \pm 13.6$ & 0.001 \\
Female, \% & 54.1 & 59.1 & 0.29 \\
Caucasian, \% & 89.2 & 85.2 & 0.22 \\
College education, \% & 46.0 & 45.3 & 0.89 \\
Married, \% & 59.6 & 53.8 & 0.23 \\
Employed, \% & 41.2 & 25.4 & 0.0005 \\
Receiving compensation for pain, \% & 18.1 & 33.1 & 0.0005 \\
Type of compensation, \% & & & 0.20 \\
$\quad$ Social security & 51.2 & 56.4 & \\
$\quad$ Long-term disability & 7.3 & 10.9 & \\
$\quad$ Workers' compensation & 14.6 & 9.1 & \\
$\quad$ Sick leave & 12.2 & 1.8 & \\
$\quad$ No-fault insurance & 14.6 & 21.8 & \\
Pain phenotype & & & \\
Pain severity (BPI) & $6.02 \pm 1.83$ & $7.2 \pm 1.54$ & $<0.0001$ \\
Pain interference (BPI) & $6.33 \pm 2.11$ & $7.95 \pm 1.69$ & $<0.0001$ \\
Neuropathic pain descriptors (PainDETECT questionnaire) & $14 \pm 7.85$ & $22.1 \pm 7.79$ & $<0.0001$ \\
Physical function (ODI) & $40.3 \pm 16.6$ & $55.3 \pm 15.8$ & $<0.0001$ \\
Physical function (PROMIS Physical Function Short Form 1) & $31.9 \pm 7.42$ & $26.9 \pm 7.81$ & $<0.0001$ \\
Anxiety (HADS) & $6.61 \pm 3.67$ & $11.7 \pm 4.65$ & $<0.0001$ \\
Depression (HADS) & $7.15 \pm 4.23$ & $11.5 \pm 4.4$ & $<0.0001$ \\
Duration of pain, \% & & & 0.0096 \\
<3 months & 8.1 & 1.0 & \\
3-6 months & 11.4 & 3.1 & \\
7-12 months & 12.2 & 12.5 & \\
1-5 years & 35.0 & 42.7 & \\
>5 years & 33.3 & 40.6 & \\
\hline
\end{tabular}

* Except where indicated otherwise, values are the mean \pm SD. The fibromyalgia (FM) criteria and severity scales score ranges from 0 to 31 . The Brief Pain Inventory (BPI) measures pain intensity (mean score of 4 questions regarding current, worst, least, and average pain intensity over last 7 days, from 0 to 10 , where $0=$ no pain and $10=$ worst pain imaginable) and pain interference (mean score of the 7 interference questions of the BPI, from 0 to 10 , where $0=$ does not interfere and $10=$ totally interferes). The PainDETECT questionnaire measures neuropathic pain on a scale of -1 to 38 , where higher scores indicate more neuropathic pain. The Oswestry Disability Index (ODI) ranges from 0 to 100, where a higher score indicates more disability. The Patient-Reported Outcomes Measurement Information System (PROMIS) Physical Function Short Form 1 ranges from 10 to 50, where lower scores indicate lower physical function. The Hospital Anxiety and Depression Scale (HADS) ranges from 0 to 21, where higher scores indicate more anxiety and more symptoms of depression.

plains that the information will be used for clinical care and research, and a waiver of written informed consent was obtained from the Institutional Review Board. The International Classification of Diseases, Ninth Revision (ICD-9) code assigned by the treating physician was used to define the spine pain cohort. Disorders of the axial neck, middle and low back, or surrounding spine structures, as well as radicular pain and results of failed spine surgery, were all included (Table 1). A 3 -month audit of the number of new patients evaluated, with phenotyping included in the data set, demonstrated that data were captured for $85.7 \%$ of the patients (data not shown).

Instruments used to evaluate patients. The FM criteria and severity scales consist of an assessment of widespread pain and symptom severity $(27,28)$. The Widespread Pain Index (WPI) was calculated using the Michigan Body Map (31) to assess the 19 specific body areas described in the FM criteria and severity scales (score 0-19). Symptom severity was evaluated using the Symptom Severity (SS) scale (score 0-12). As per the FM survey criteria, patients were classified as FM positive if their scores were WPI $\geq 7$ and SS $\geq 5$ or WPI 3-6 and $\mathrm{SS} \geq 9$. The validity of the FM criteria and severity scales has been established, both when compared with the 1990 ACR classification criteria (which include the tender point examination) $(29,32)$ and when compared with the 2010 ACR preliminary diagnostic criteria (which include physician's assessment) $(28,33)$. Patients missing FM criteria and severity scales data were excluded from analysis. Additional phenotyping measures were completed/recorded, including the Brief Pain Inventory (BPI) (pain intensity and interference) (34), the PainDETECT questionnaire (neuropathic pain) (35), the Oswestry Disability Index (ODI) (physical function) (36), the Patient-Reported Outcomes Measurement Information System (PROMIS) Physical Function Short Form 1 (physical function) (37), the Hospital Anxiety and Depression Scale (HADS) (symptoms of depression and anxiety) (38), and the duration of pain.

Statistical analysis. Data were entered into the Assessment of Pain Outcomes Longitudinal Electronic Data Capture system (30). Missing data for the validated instruments were handled as described by authors of the instruments $(35,38,39)$. 
Table 3. Multivariate analysis of the association between pain and phenotypic variables and FM status*

\begin{tabular}{lccc}
\hline \multicolumn{1}{c}{ Model, predictor variable } & Estimate & SE & $P$ \\
\hline Linear & & & \\
$\quad$ Intercept & 2.711 & 0.765 & 0.0004 \\
Sex (male vs. female) & -1.567 & 0.422 & 0.0002 \\
Physical function (ODI) & 0.028 & 0.018 & 0.12 \\
$\quad$ Pain interference (BPI) & 0.432 & 0.152 & 0.0047 \\
$\quad$ Neuropathic pain (PainDETECT & 0.129 & 0.030 & $<0.0001$ \\
$\quad$ questionnaire) & & & \\
$\quad$ Anxiety (HADS) & 0.412 & 0.051 & $<0.0001$ \\
Logistic & & & \\
Intercept & -4.672 & 0.518 & $<0.0001$ \\
$\quad$ Neuropathic pain (PainDETECT & 0.069 & 0.018 & 0.0002 \\
$\quad$ questionnaire) & & & \\
$\quad$ Physical function (ODI) & 0.021 & 0.009 & 0.024 \\
$\quad$ Anxiety (HADS) & 0.221 & 0.034 & $<0.0001$ \\
Compensation for pain & 0.485 & 0.299 & 0.11 \\
\hline
\end{tabular}

* Measured as a continuous score (linear regression) or dichotomized (logistic regression) using the FM criteria and severity scales. The best models after variable selection are presented. See Table 2 for definitions.

As noted above, patients who did not complete all the components of the FM criteria and severity scales were not included in the analysis. Additionally, patients missing more than 1 item on the BPI subscales, PainDETECT questionnaire, and ODI were excluded. The PROMIS Physical Function Short Form 1 requires complete data on all 10 items, so patients missing any items were excluded. For the HADS, when 6 of the 7 questions were answered, a single value for the missing item was inferred by imputation of the mean of the other 6 values as recommended. For the other instruments, only 1 missing question was allowed; however, other completed questionnaires were allowed (i.e., patients were not completely excluded from the analysis for having 1 incomplete questionnaire).

Data were analyzed using $\mathrm{R}$ software, version 2.15.0 (www.r-project.org) and SPSS software, version 19. Betweengroup comparisons were performed using a $t$-test for continuous variables, a chi-square test for binary variables, and a Wilcoxon test for ordered categorical variables. The significance level was set at 0.025 to account for the previous analysis on a smaller data set conducted for the presented abstract. We studied patterns of association between the observed phenotype, and their relationship with the centralized pain phenotype, using binary categorization of FM status by the previously described FM criteria and severity scales (FM positive versus FM negative) $(27,28)$ and the continuous "fibromyalgianess" score (the sum of the WPI and SS scales). Multivariate linear regression was used to study association between the continuous FM score and measured phenotype and pain variables. Association of the phenotype and pain variable panel with the binary FM status was analyzed using logistic regression. Best model selection using Akaike's information criterion (AIC) and likelihood ratio tests was used to select the best set of predictor variables. Prediction strength of the logistic model was analyzed using a receiver operating characteristic (ROC) curve. The ROC curve analysis was crossvalidated (10-fold) to ensure reproducibility of predictions and avoid model overfitting. Cross-validated estimate of the area under the ROC curve ( $\mathrm{C}$ statistic) was used to characterize the predictive performance of the phenotype and pain profile with respect to the FM categorization (FM positive or FM negative). Model adequacy was tested using a variety of standardized residual plots (not shown).

Radicular pain and spinal stenosis are frequently associated with neuropathic pain symptoms and are often differentiated from axial spine pain. In order to specifically analyze the portion of the cohort with axial spine pain (neck pain, middle back pain, and low back pain), the multivariate analyses noted above were repeated after excluding patients with radicular or non-axial spine pain disorders (excluded ICD-9 codes 723.4 [cervical radiculopathy], 721.1 [cervical spondylosis], 722.81 [postlaminectomy syndrome cervical], 723 [cervical spinal stenosis], 722.11 [thoracic herniated nucleus pulposus], 722.1 [lumbar herniated nucleus pulposus], 724.4 [thoracic or lumbar radiculopathy], 724.02 [lumbar spinal stenosis], 722.83 [lumbar postlaminectomy syndrome], 739.4 [sacroiliac joint dysfunction], 720.2 [sacroiliitis]; total $\mathrm{n}=130$ ) (Table 1).

\section{RESULTS}

A total of 1,208 new patients were seen in the pain clinic over the defined time period, with diagnoses of primary spine pain in 548 patients. After exclusion of patients who did not have complete data for the FM criteria and severity scales, 443 patients were retained for the analysis (Table 2). Of these patients, 186 $(42 \%)$ were FM positive according to the FM criteria and severity scales. In univariate analyses, FM-positive patients were younger $(P=0.001)$, less likely to be employed $(P=0.0005)$, and more likely to be receiving compensation for their pain $(P=0.0005)$, but there were no other significant differences between the 2 groups with respect to demographic variables (Table 2). Compared to FM-negative patients, FM-positive patients were more likely to have pain of a longer duration $(P=$ 0.0096) and had higher scores on measures of pain severity, pain interference, and neuropathic pain $(P<$ 0.0001). FM-positive patients also reported lower physical function and showed higher levels of anxiety and more symptoms of depression $(P<0.0001)$.

The multivariate analyses of the associations between the observed demographic and pain/mood/ function phenotype and both the continuous FM score and the binary FM classification are shown in Table 3 . FM status (positive versus negative) could be best predicted using neuropathic pain (the PainDETECT questionnaire), physical function (the ODI), anxiety (the HADS), and compensation for pain, with a high area under the ROC curve ( $\mathrm{C}$ statistic, area under the curve $[$ AUC $]=0.80)$. The best model search using AIC resulted in the same model as the cross-validated ROC 


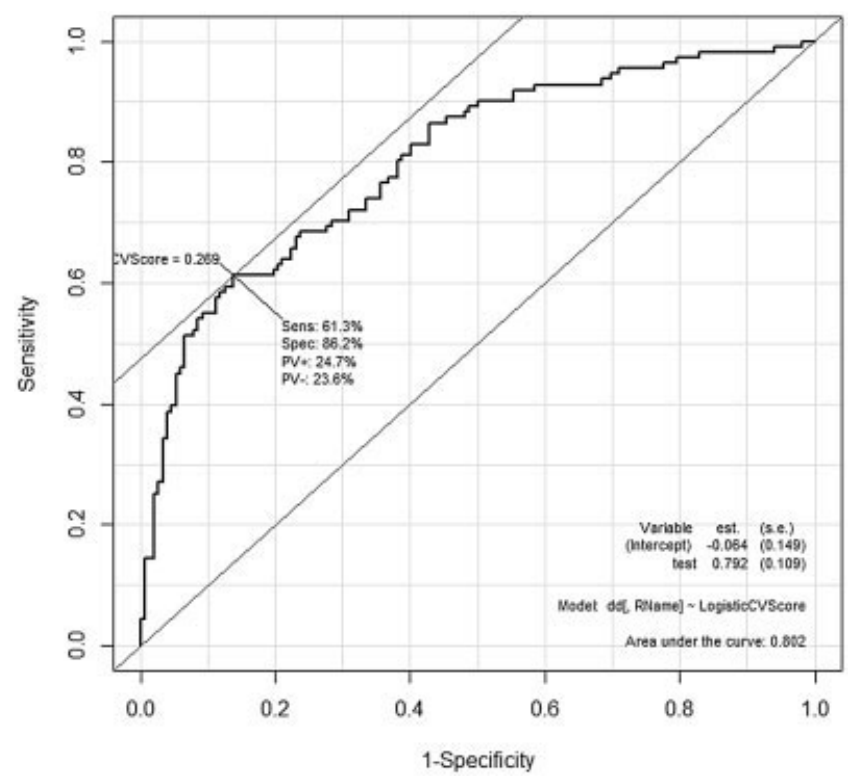

Figure 1. Receiver operating characteristic curve for predicting fibromyalgia (FM) status (according to the FM criteria and severity scales) using a panel of demographic, pain, and other phenotypic predictor variables. $\mathrm{CV}=$ cross-validated; Sens. = sensitivity; Spec. = specificity; $\mathrm{PV}+=$ positive predictive value; $\mathrm{PV}-=$ negative predictive value; est. $=$ estimate; s.e. $=$ standard error; $\mathrm{dd}[$, $\mathrm{RName}]=$ response $(\mathrm{FM}+$ vs. $\mathrm{FM}-)$.

curve analysis (Figure 1). Multivariate prediction of the FM score as a continuous score reported a similar set of highly significant best model predictors: sex (male versus female), pain interference (the BPI), neuropathic pain, and anxiety $(P<0.005$ for each significant predictor $)$ (Table 3).

A subanalysis of the portion of the cohort with axial spine pain was conducted, which resulted in a reduction in the number of patients $(n=418)$ available for the analysis (see statistical analysis section in Patients and Methods for additional details). Descriptive betweengroup analysis in the subset of patients with axial spine pain yielded an outcome very similar to that shown in Table 1 (not shown). The smaller sample size resulted in a reduced panel of pain and phenotype variables in the trimmed multivariate models. Pain interference, neuropathic pain, and anxiety were the best predictors after variable selection using linear (continuous FM status) and logistic (binary FM status) models $(P<0.001$ for all variables). The analysis yielded almost the same AUC (C statistic) of 0.81 (not shown).

\section{DISCUSSION}

We found that $42 \%$ of the patients presenting to a tertiary care facility with a primary diagnosis of spine pain had FM according to the FM criteria and severity scales, which suggests the presence of centralized pain. To our knowledge, this is the first time that the FM criteria and severity scales have been used to differentiate a spine pain cohort. FM-positive patients reported a strikingly different pain phenotype from FM-negative patients (i.e., in univariate analyses the former were more likely to be female, younger, unemployed, receiving compensation for their pain, reporting greater pain intensity and interference, anxious, depressed, using neuropathic pain descriptors, and reporting diminished functioning) (Table 2). Analyzing the groups using the FM criteria and severity scales score as a dichotomous (logistic) or continuous (linear) measure further demonstrated that neuropathic pain descriptors, anxiety, pain interference, and physical function were independently predictive of the FM criteria and severity scales score (Table 3), which is consistent with what has previously been described in patients diagnosed as having FM using the 1990 ACR classification criteria (32). The independent predictors from the multivariate models are some of the most commonly described predictors of poor outcomes in minimally invasive spine interventions and postsurgical pain. Hence, there may be a common underlying pathophysiology or "diagnosis" driving these findings.

The diagnosis of FM has been a point of controversy for years in the community of pain specialists, as there is no definitive diagnostic test. FM positivity according to the FM criteria and severity scales does not confirm categorical diagnosis. Instead, according to the 2012 Canadian Guidelines for the diagnosis and management of fibromyalgia syndrome (http://www.canadian painsociety.ca/pdf/Fibromyalgia_Guidelines_2012.pdf), the taking of a history, a physical examination, and laboratory testing (e.g., blood count, erythrocyte sedimentation rate, C-reactive protein, creatine kinase, and thyroid-stimulating hormone) by an experienced provider are required to make the diagnosis of FM. However, comorbid FM is common in nearly all other musculoskeletal chronic pain conditions, including osteoarthritis and rheumatoid arthritis (estimates range from $20 \%$ to $30 \%)(23,24)$. The validity of the FM criteria and severity scales has been established, both when compared with the 1990 ACR classification criteria (which include the tender point examination) $(29,32)$ and when compared with the 2010 ACR preliminary diagnostic criteria (which include physician's assessment) $(28,33)$. As was previously recommended, the present study applied this validated self-report measure in an epidemiologic manner to detect widespread body pain and comorbid symptoms. 
These data and those from experimental studies $(26,40,41)$ suggest that centralized pain may be very common in spine pain. For example, 2 studies have shown that a sizable proportion of individuals with chronic low back pain display diffuse tenderness (e.g., mechanical hyperalgesia) and have functional magnetic resonance imaging findings consistent with FM or similar centralized pain states $(25,26)$. Using the 4-quadrant pain described in the 1990 ACR classification criteria for FM, a retrospective study showed that female patients with higher pain severity, a family history of chronic widespread pain, and more painful comorbidities were more likely to transition from neck and back pain to chronic widespread pain (42). In the present study, independent predictors of FM positivity according to the FM criteria and severity scales included female sex, higher levels of neuropathic pain descriptors, anxiety, more pain interference, and lower levels of physical function (Table 3). Neuropathic pain descriptors in particular are thought to be strongly associated with the FM pain phenotype and have been shown to correlate with the number of tender points and pain sensitivity during experimental pain testing $(20,21)$. Because radicular pain would be expected to present with more neuropathic pain symptoms, the analyses were also repeated after excluding radicular pain and non-axial spine pain diagnoses, and the results did not change.

Most axial spine pain diagnoses (e.g., facet arthropathy, lumbago) are not normally thought to be associated with high levels of neuropathic pain, yet among FM-positive patients according to the FM criteria and severity scales, the mean value for the PainDETECT measure exceeded the instrument's defined cut point for neuropathic pain -with or without including patients with radicular pain diagnoses (35). Although patients in different diagnostic groups who meet the criteria for neuropathic pain using the PainDETECT questionnaire certainly describe similar levels of pain, the phenotype of FM-positive patients according to the FM criteria and severity scales is distinct.

The finding that many patients with spine pain report widespread body pain and comorbid symptoms, such as fatigue, trouble thinking, anxiety, and/or depression, could have important treatment implications and may explain a portion of the high failure rates described for some of the most common spine interventions. Injections and peripherally targeted analgesics would be expected to provide less benefit in a patient with altered central pain processing than in those with predominantly peripheral pathology. FM is associated with lower levels of neurotransmitters that inhibit pain, including norepinephrine, serotonin, and $\gamma$-aminobutyric acid, along with higher levels of neurotransmitters that increase pain, including glutamate $(15,43)$. FM patients also have lower endogenous opioid receptor binding availability and high levels of cerebrospinal fluid opioids (44), together suggesting that the endogenous opioid system is already activated in FM and thus perhaps explaining the anecdotal experience that opioids are ineffective in most of these patients (45).

The use of minimally invasive interventions for spine pain, such as epidural steroid injections and facet joint interventions, has increased dramatically in recent years (3). A recent well-publicized meta-analysis has called into question the long-term efficacy of epidural steroid injections (46), and studies of response prediction estimate rates of treatment failure to be between $25 \%$ and $45 \%$ (47-49). Similarly, although the efficacy of facet joint interventions has been established in tightly selected patient populations $(50,51)$, higher failure rates $(39-47 \%)$ have been shown in retrospective studies $(5,6)$, and effectiveness in standard clinical care is not known. Back and neck pain are frequently described in FM, and it is possible that the high failure rate for these interventions is in part due to intervention on a peripheral target in the spine when the nature of the patient's pain is due at least in part to brain and spinal cord dysfunction. Taken together, these data make a compelling case for the study of a modified treatment approach. For example, previous studies have demonstrated efficacy of serotonin-norepinephrine reuptake inhibitors in chronic low back pain (52). Nonpharmacologic interventions, such as cognitive behavioral therapy and exercise, have also demonstrated excellent effect sizes that often exceed those of pharmacologic interventions in FM and other pain states (53).

This study was conducted at a single large, tertiary care pain clinic, and these results may not be generalizable. The present study is largely hypothesis generating as it is cross-sectional in nature. Despite the profound phenotypic differences demonstrated, additional research is needed to evaluate the presence of centralized pain (e.g., quantitative sensory testing, neuroimaging) and then to better understand the impact of centralized pain on treatment outcomes. Prospective studies are required to examine whether some of the outcome measures used herein are better than a detailed history and physical examination and review of radiographic findings at predicting which patients with axial pain will respond best to peripherally directed procedures, regardless of "diagnosis" (e.g., lumbago, facet joint arthropathy). As with any data set, this study was limited by the variables included in the patientcompleted phenotype assessment. 
New patients presenting to a tertiary care pain clinic with a diagnosis of primary spine pain are commonly determined to have FM according to the FM criteria and severity scales, indicating that more widespread pain is present along with a constellation of associated symptoms that are presumed to be largely due to alterations in central neurotransmission. In the present study, patients with spine pain who were FM positive according to the FM criteria and severity scales described profound phenotypic differences from those who were FM negative, including more neuropathic pain descriptors, higher levels of anxiety symptoms, greater pain interference, and lower physical functioning. These factors largely overlap with those found to be predictive of poor outcomes in spine pain interventions. It is possible that a simple self-report measure could aid in the prediction of outcomes of some of the most common minimally invasive spine interventions.

\section{ACKNOWLEDGMENTS}

We thank David A. Williams, PhD (Professor, Department of Anesthesiology, University of Michigan, Ann Arbor) and Kevin K. Tremper, PhD, MD (Professor and Chairman, Department of Anesthesiology, University of Michigan, Ann Arbor) for guidance and support.

\section{AUTHOR CONTRIBUTIONS}

All authors were involved in drafting the article or revising it critically for important intellectual content, and all authors approved the final version to be published. Dr. Brummett had full access to all of the data in the study and takes responsibility for the integrity of the data and the accuracy of the data analysis.

Study conception and design. Brummett, Goesling, Tsodikov, Meraj, Wasserman, Clauw, Hassett.

Acquisition of data. Brummett, Goesling, Meraj, Wasserman, Hassett. Analysis and interpretation of data. Brummett, Goesling, Tsodikov, Meraj, Wasserman, Clauw, Hassett.

\section{REFERENCES}

1. United States Bone and Joint Initiative. Spine: low back and neck pain. In: The burden of musculoskeletal diseases in the United States. Prevalence, societal and economic cost. 2nd ed. Rosemont (IL): American Academy of Orthopaedic Surgeons; 2011. p. 21-56.

2. Katz JN. Lumbar disc disorders and low-back pain: socioeconomic factors and consequences. J Bone Joint Surg Am 2006;88 Suppl $2: 21-4$.

3. Manchikanti L, Pampati V, Singh V, Boswell MV, Smith HS, Hirsch JA. Explosive growth of facet joint interventions in the Medicare population in the United States: a comparative evaluation of 1997, 2002, and 2006 data. BMC Health Serv Res 2010; 10:84.

4. Manchikanti L, Pampati V, Boswell MV, Smith HS, Hirsch JA. Analysis of the growth of epidural injections and costs in the Medicare population: a comparative evaluation of 1997, 2002, and 2006 data. Pain Physician 2010;13:199-212.

5. Cohen SP, Bajwa ZH, Kraemer JJ, Dragovich A, Williams KA,
Stream J, et al. Factors predicting success and failure for cervical facet radiofrequency denervation: a multi-center analysis. Reg Anesth Pain Med 2007;32:495-503.

6. Cohen SP, Stojanovic MP, Crooks M, Kim P, Schmidt RK, Shields $\mathrm{CH}$, et al. Lumbar zygapophysial (facet) joint radiofrequency denervation success as a function of pain relief during diagnostic medial branch blocks: a multicenter analysis. Spine J 2008;8:498-504.

7. Cohen SP, Hurley RW, Christo PJ, Winkley J, Mohiuddin MM, Stojanovic MP. Clinical predictors of success and failure for lumbar facet radiofrequency denervation. Clin J Pain 2007;23:45-52.

8. Stojanovic MP, Sethee J, Mohiuddin M, Cheng J, Barker A, Wang J, et al. MRI analysis of the lumbar spine: can it predict response to diagnostic and therapeutic facet procedures? Clin J Pain 2010;26:110-5.

9. Manchikanti L, Cash KA, Pampati V, Fellows B. Influence of psychological variables on the diagnosis of facet joint involvement in chronic spinal pain. Pain Physician 2008;11:145-60.

10. Schiff E, Eisenberg E. Can quantitative sensory testing predict the outcome of epidural steroid injections in sciatica? A preliminary study. Anesth Analg 2003;97:828-32.

11. Revel M, Poiraudeau S, Auleley GR, Payan C, Denke A, Nguyen $\mathrm{M}$, et al. Capacity of the clinical picture to characterize low back pain relieved by facet joint anesthesia: proposed criteria to identify patients with painful facet joints. Spine 1998;23:1972-6.

12. Revel ME, Listrat VM, Chevalier XJ, Dougados M, N'guyen MP, Vallee C, et al. Facet joint block for low back pain: identifying predictors of a good response. Arch Phys Med Rehabil 1992;73: $824-8$.

13. Wylde V, Hewlett S, Learmonth ID, Dieppe P. Persistent pain after joint replacement: prevalence, sensory qualities, and postoperative determinants. Pain 2011;152:566-72.

14. Woolf CJ. Central sensitization: implications for the diagnosis and treatment of pain. Pain 2011;152:S2-15.

15. Williams DA, Clauw DJ. Understanding fibromyalgia: lessons from the broader pain research community. J Pain 2009;10 777-91.

16. Clauw DJ, Schmidt M, Radulovic D, Singer A, Katz P, Bresette J. The relationship between fibromyalgia and interstitial cystitis J Psychiatr Res 1997;31:125-31.

17. Sarzi-Puttini P, Atzeni F, Mease PJ. Chronic widespread pain: from peripheral to central evolution. Best Pract Res Clin Rheumatol 2011;25:133-9.

18. El-Gabalawy H, Ryner L. Central nervous system abnormalities in fibromyalgia: assessment using proton magnetic resonance spectroscopy. J Rheumatol 2008;35:1242-4.

19. Schmidt-Wilcke T, Clauw DJ. Pharmacotherapy in fibromyalgia (FM) - implications for the underlying pathophysiology. Pharmacol Ther 2010;127:283-94.

20. Amris K, Jespersen A, Bliddal H. Self-reported somatosensory symptoms of neuropathic pain in fibromyalgia and chronic widespread pain correlate with tender point count and pressure-pain thresholds. Pain 2010;151:664-9.

21. Rehm SE, Koroschetz J, Gockel U, Brosz M, Freynhagen R, Tolle TR, et al. A cross-sectional survey of 3035 patients with fibromyalgia: subgroups of patients with typical comorbidities and sensory symptom profiles. Rheumatology (Oxford) 2010;49:1146-52.

22. Fitzcharles MA, Yunus MB. The clinical concept of fibromyalgia as a changing paradigm in the past 20 years. Pain Res Treat 2012; 2012:184835

23. Clauw DJ, Witter J. Pain and rheumatology: thinking outside the joint [editorial]. Arthritis Rheum 2009;60:321-4.

24. Lee YC, Nassikas NJ, Clauw DJ. The role of the central nervous system in the generation and maintenance of chronic pain in rheumatoid arthritis, osteoarthritis and fibromyalgia. Arthritis Res Ther 2011;13:211.

25. Clauw DJ, Williams D, Lauerman W, Dahlman M, Aslami A, Nachemson AL, et al. Pain sensitivity as a correlate of clinical status in individuals with chronic low back pain. Spine 1999;24: 2035-41. 
26. Giesecke T, Gracely RH, Grant MA, Nachemson A, Petzke F, Williams DA, et al. Evidence of augmented central pain processing in idiopathic chronic low back pain. Arthritis Rheum 2004;50: $613-23$.

27. Wolfe F, Clauw DJ, Fitzcharles MA, Goldenberg DL, Hauser W, Katz RS, et al. Fibromyalgia criteria and severity scales for clinical and epidemiological studies: a modification of the ACR Preliminary Diagnostic Criteria for Fibromyalgia. J Rheumatol 2011;38: 1113-22.

28. Wolfe F, Clauw DJ, FitzCharles MA, Goldenberg DL, Katz RS, Mease P, et al. The American College of Rheumatology preliminary diagnostic criteria for fibromyalgia and measurement of symptom severity. Arthritis Care Res (Hoboken) 2010;62:600-10.

29. Hauser W, Jung E, Erbsloh-Moller B, Gesmann M, Kuhn-Becker $\mathrm{H}$, Petermann F, et al. Validation of the Fibromyalgia Survey Questionnaire within a cross-sectional survey. PLoS One 2012;7: e37504.

30. Hassett AL, Wasserman R, Goesling J, Rakovitis K, Shi B, Brummett CM. Longitudinal assessment of pain outcomes in the clinical setting: development of the "APOLO" Electronic Data Capture system. Reg Anesth Pain Med 2012;37:398-402.

31. Brummett CM, Hassett AL, Brummett KA, Clauw DJ, Williams DA. The Michigan Body Map and its use in assessing the American College of Rheumatology survey criteria for fibromyalgia [abstract]. Arthritis Rheum 2011;63 Suppl:S368.

32. Wolfe F, Smythe HA, Yunus MB, Bennett RM, Bombardier C, Goldenberg DL, et al. The American College of Rheumatology 1990 criteria for the classification of fibromyalgia: report of the Multicenter Criteria Committee. Arthritis Rheum 1990;33:160-72.

33. Vincent A, Lahr BD, Wolfe F, Clauw DJ, Whipple MO, Oh TH, et al. Prevalence of fibromyalgia: a population-based study in Olmsted County, Minnesota, utilizing the Rochester Epidemiology Project. Arthritis Care Res (Hoboken) 2013;65:786-92.

34. Tan G, Jensen MP, Thornby JI, Shanti BF. Validation of the Brief Pain Inventory for chronic nonmalignant pain. J Pain 2004; 5:133-7.

35. Freynhagen R, Baron R, Gockel U, Tolle TR. painDETECT: a new screening questionnaire to identify neuropathic components in patients with back pain. Curr Med Res Opin 2006;22:1911-20.

36. Fairbank JC, Pynsent PB. The Oswestry Disability Index. Spine 2000;25:2940-52

37. Cella D, Riley W, Stone A, Rothrock N, Reeve B, Yount S, et al, on behalf of the PROMIS Cooperative Group. The PatientReported Outcomes Measurement Information System (PROMIS) developed and tested its first wave of adult self-reported health outcome item banks: 2005-2008. J Clin Epidemiol 2010;63:1179-94.

38. Zigmond AS, Snaith RP. The Hospital Anxiety and Depression Scale. Acta Psychiatr Scand 1983;67:361-70.

39. Keller S, Bann CM, Dodd SL, Schein J, Mendoza TR, Cleeland
CS. Validity of the Brief Pain Inventory for use in documenting the outcomes of patients with noncancer pain. Clin J Pain 2004; 20:309-18.

40. George SZ, Wittmer VT, Fillingim RB, Robinson ME. Sex and pain-related psychological variables are associated with thermal pain sensitivity for patients with chronic low back pain. J Pain 2007;8:2-10.

41. Puta C, Schulz B, Schoeler S, Magerl W, Gabriel B, Gabriel HH, et al. Enhanced sensitivity to punctate painful stimuli in female patients with chronic low back pain. BMC Neurol 2012;12:98.

42. Kindler LL, Jones KD, Perrin N, Bennett RM. Risk factors predicting the development of widespread pain from chronic back or neck pain. J Pain 2010;11:1320-8.

43. Harris RE, Sundgren PC, Craig AD, Kirshenbaum E, Sen A, Napadow V, et al. Elevated insular glutamate in fibromyalgia is associated with experimental pain. Arthritis Rheum 2009;60: 3146-52.

44. Harris RE, Clauw DJ, Scott DJ, McLean SA, Gracely RH, Zubieta JK. Decreased central $\mu$-opioid receptor availability in fibromyalgia. J Neurosci 2007;27:10000-6.

45. Mease PJ, Dundon K, Sarzi-Puttini P. Pharmacotherapy of fibromyalgia. Best Pract Res Clin Rheumatol 2011;25:285-97.

46. Pinto RZ, Maher CG, Ferreira ML, Hancock M, Oliveira VC, McLachlan AJ, et al. Epidural corticosteroid injections in the management of sciatica: a systematic review and meta-analysis. Ann Intern Med 2012;157:865-77.

47. Iversen T, Solberg TK, Romner B, Wilsgaard T, Twisk J, Anke A et al. Effect of caudal epidural steroid or saline injection in chronic lumbar radiculopathy: multicentre, blinded, randomised controlled trial. BMJ 2011;343:d5278.

48. Sayegh FE, Kenanidis EI, Papavasiliou KA, Potoupnis ME, Kirkos JM, Kapetanos GA. Efficacy of steroid and nonsteroid caudal epidural injections for low back pain and sciatica: a prospective, randomized, double-blind clinical trial. Spine 2009;34:1441-7.

49. Wilkinson IM, Cohen SP. Epidural steroid injections. Curr Pain Headache Rep 2012;16:50-9.

50. Dreyfuss P, Halbrook B, Pauza K, Joshi A, McLarty J, Bogduk N. Efficacy and validity of radiofrequency neurotomy for chronic lumbar zygapophysial joint pain. Spine 2000;25:1270-7.

51. Lord SM, Barnsley L, Wallis BJ, McDonald GJ, Bogduk N. Percutaneous radio-frequency neurotomy for chronic cervical zygapophyseal-joint pain. N Engl J Med 1996;335:1721-6.

52. Skljarevski V, Desaiah D, Liu-Seifert H, Zhang Q, Chappell AS, Detke MJ, et al. Efficacy and safety of duloxetine in patients with chronic low back pain. Spine (Phila Pa 1976) 2010;35:E578-85.

53. Glombiewski JA, Sawyer AT, Gutermann J, Koenig K, Rief W, Hofmann SG. Psychological treatments for fibromyalgia: a metaanalysis. Pain 2010;151:280-95. 